\title{
Поляризация фотолюминесценции квантовых точек, внедренных в квантовые нити
}

\author{
() А.В. Платонов*, В.П. Кочерешко*+, В.Н. Кац*, Г.Э. Цырлин*, А.Д. Буравлев*, \\ Д.В. Авдошина*, A. Delga ${ }^{\bullet}$, L. Besombes ${ }^{\bullet}$, H. Mariette ${ }^{\bullet}$ \\ * Физико-технический институт им. А.Ф. Иоффе Российской академии наук, \\ 194021 Санкт-Петербург, Россия \\ + Санкт-Петербургский государственный университет, \\ 199034 Санкт-Петербург, Россия \\ -CEA-CNRS group „Nanophysique et Semiconducteurs“, \\ CEA, INAC, SP2M, and Institut Néel, \\ 17 rue des Martyrs, F-38054 Grenoble, France \\ E-mail: alexei.platonov@mail.ioffe.ru
}

(Получена 27 апреля 2016 г. Принята к печати 10 мая 2016 г.)

\begin{abstract}
Исследовались спектры фотолюминесценции одиночных квантовых точек, заключенных в квантовую нить. Из поведения спектров в магнитном поле удалось оценить энергию связи экситона в квантовой точке, заключенной в квантовую нить. Обнаружено, что сигнал экситонной фотолюминесценции из квантовой точки, излучаемый в направлении оси нанонити, поляризован линейно. В то же время сигнал фотолюминесценции, распространяющийся в направлении, перпендикулярном оси нанонити, практически не был поляризован. Наблюдаемый эффект объяснен неаксиальным расположением точки относительно оси нити в условиях гигантского увеличения энергии связи экситона, связанного с влиянием на экситон потенциала изображения.
\end{abstract}

\section{1. Введение}

Несмотря на возрастающий интерес, квазиодномерные структуры с квантовыми нитями пока остаются слабо исследованными. До последнего времени удовлетворительной технологии получения таких структур не существовало. Совсем недавно появился метод выращивания структур с квантовыми нитями с использованием металлического катализатора. За несколько прошедших лет технология изготовления таких структур была значительно усовершенствована. В настоящее время изготовление и исследование структур с квантовыми нитями является быстро развивающейся областью физики полупроводниковых наноструктур. Наблюдается „взрывной“ характер роста числа публикаций на эту тему. Появились и специализированные международные конференции, посвященные физике полупроводниковых квантовых нитей.

Современный уровень развития метода каталитического MBE-роста, который продолжает активно развиваться, позволяет получать образцы высочайшего качества, в том числе выращивать внутри одиночных нанонитей одну или несколько квантовых точек геометрически правильной формы. Квантовые точки, выращенные внутри нанонитей, имеют ряд преимуществ перед самоорганизованными квантовыми точками, выращенными методом Странского-Крастанова, имея значительно больший квантовый выход, а также обладая естественными электрическими контактами. Последнее свойство позволяет исследовать электрооптические свойства точек. Нанонить с квантовой точкой внутри способна стать источником запутанных фотонов, что может быть использовано в криптографии и квантовых вычислениях. Интерес исследователей к квантовым нитям обусловлен не только новизной самого объекта, но и стремлением обнаружить новые физические явления в квазиодномерных структурах.

\section{2. Эксперимент}

Изучались нанонити (HH) на основе AlGaAs, содержащие GaAs квантовые точки (КТ) внутри нити. Нанонити выращивались на полуизолирующих подложках GaAs (111) в EP1203 MBE системе, оборудованной твердыми источниками $\mathrm{Ga}$ и $\mathrm{Al}$ и дополнительной ячейкой мышьяка. При данной ориентации подложки НН растут строго перпендикулярно поверхности.

Характеризация полученных образцов проводилась с помощью сканирующего электронного микроскопа (СЭМ) и просвечивающего электронного микроскопа (ПЭМ). Исследованные КТ имеют вид дисков, выращенных из GaAs, окруженных полупроводником с более широкой запрещенной зоной $\mathrm{AlGaAs}$. Диаметр $\mathrm{HH}$ в наших образцах составлял 20-50 нм, длина НН 500 нм, толщина КТ была несколько атомных монослоев, т.е. $2-5$ нм. Детальное описание структур приведено в работах $[1,2]$.

Исследовались спектры ФЛ при непрерывном возбуждении и с временны́м разрешением при температурах в диапазоне от 5 до $250 \mathrm{~K}$ как функция уровня оптического возбуждения.

В экспериментах с импульсным возбуждением образцы возбуждались второй гармоникой $\mathrm{Nd}$ :YAG-лазера или удвоенного Ti:Sapphire-лазера с длительностью пульса 100 фс и частотой повторения 70 МГц. Средняя мощность лазеров была приблизительно 10 мВт. В экспериментах с непрерывным возбуждением использовался лазер на красителях, накачиваемый второй гармоникой $\mathrm{Nd}$ :YAG-лазера. 
Монохроматор Jobin-Yvon 50 с ПЗС детектором использовался для регистрации спектров. Для измерения спектров микрофотолюминесценции одиночных НН был использован микроскоп для фокусировки лазерного луча и сбора сигнала. Минимальный размер лазерного пятна на образце составлял 1.5 мкм, что позволило нам выделить сигнал от одиночной нити. Кроме того, установка позволяла прикладывать магнитные поля до 8 Тл.

\section{3. Результаты}

На рис. 1 представлен спектр фотолюминесценции (ФЛ), снятый от одиночной квантовой точки, помещенной в квантовую нить. При малых интенсивностях оптического возбуждения, $\sim 1 \mathrm{BT} / \mathrm{cm}^{2}$, спектр представляет собой узкую линию на энергии 1.653 эВ с шириной порядка 0.2 мэВ. Эта ширина превосходит разрешающую способностью спектрометра и заведомо больше однородной ширины линии излучения КТ. Ширина линии связана со спектральной диффузией и детально проанализирована в работе [3]. Эту линию в спектре ФЛ естественно связать с аннигиляцией экситона в основном состоянии из одиночной КТ. На том же рисунке приведен спектр возбуждения фотолюминесценции (ВФЛ) от той же точки с регистрацией на линии основного состояния экситона. Спектр состоит из набора относительно узких линий, связанных с поглощением света возбужденными состояниями экситона в квантовой точке и последующим излучением из основного состояния. В этом спектре расстояние между линиями поглощения определяется „поперечным“ (перпендикулярным оси роста) квантованием носителей в квантовой точке диаметром $\sim 15-25$ нм. Энергия „продольного“ квантования носителей (вдоль оси роста) в квантовой точке составляет $\sim 130$ мэВ, что соответствует толщине точки в 2.5 нм.

С ростом интенсивности возбуждения линия излучения экситона в спектре ФЛ быстро насыщалась и в

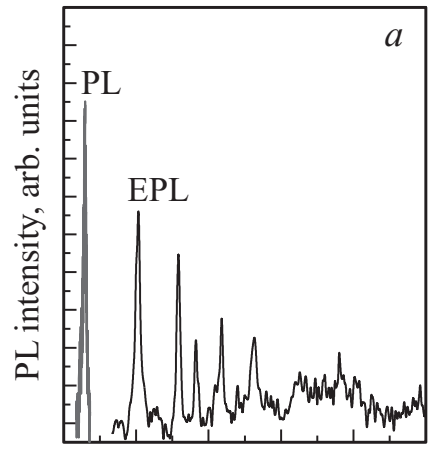

1.661 .671 .681 .69

Energy, eV

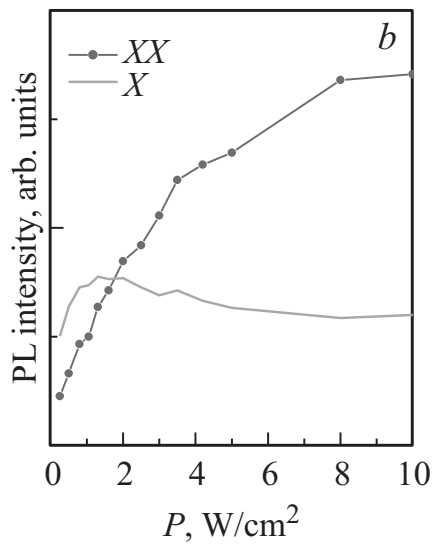

Рис. 1. $a-$ спектр ФЛ и возбуждения ФЛ от одиночной КТ в отдельной нанонити, высота КТ $2.5 \mathrm{Hм}, T=6 \mathrm{~K} . b-$ зависимость интегральной интенсивности ФЛ одиночной КТ для линий экситона $(X)$ и биэкситона $(X X)$ от интенсивности накачки.

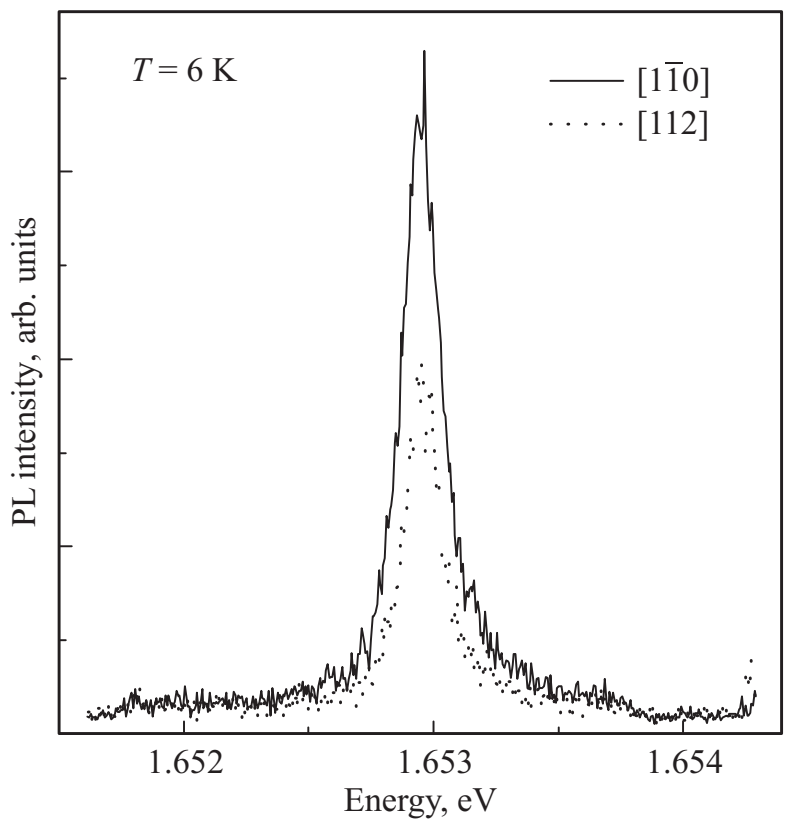

Рис. 2. Спектры поляризованной ФЛ из одиночной КТ при излучении вдоль оси роста [111]. Сплошная кривая - поляризация вдоль [11이. Пунктир - поляризация вдоль [112].

спектре появлялась еще одна линия, сдвинутая на 8 мэВ в сторону меньших энергий. Эту линию естественно связать с рекомбинацией биэкситона. На рис. $1, b$ представлена зависимость интенсивности линии излучения экситона $(X)$ от интенсивности возбуждения $(P)$. Видно, что линия экситона быстро насыщается и в спектре начинает доминировать линия излучения биэкситона $(X X)$. Насыщение экситонной линии происходило уже при интенсивностях возбуждения в несколько Вт/см ${ }^{2}$. Максимальная интенсивность излучения одиночной квантовой точки ограничена временем жизни экситона, которое составляет $\sim 4$ наносекунды в наших структурах [1]. Следовательно, линия излучения экситона должна насыщаться при интенсивностях возбуждения порядка нескольких Вт/см ${ }^{2}$ даже в предположении, что в процессе излучательной рекомбинации экситона в КТ участвует только каждый сотый фотон, поглощенный в наноните.

В исследованных структурах было обнаружено, что излучение в направлении [111] поляризовано линейно в направлении кристаллографической оси [110] или перпендикулярной ей, оси [1112] (рис. 2). Это выглядит удивительно потому, что ось роста нитей совпадает кристаллографической осью 3-го порядка (111), обладающей высокой симметрией $C_{3 v}$ для двух возможных кристаллических фаз (вюрцита и цинковой обманки) [2]. В этом направлении в силу симметрии структуры никакой линейной поляризации быть не может. Тем не менее было обнаружено, что при регистрации ФЛ вдоль оси [111] сигнал ФЛ оказывается линейно поляризованным вдоль осей $[110]$ или [1ํㅡㄹ и величина поляризации менялась от образца к образцу и могла достигать $80 \%$.

В спектре ФЛ ,лежащей“ нанонити было обнаружено еще одно неожиданное явление. Было обнаружено, 


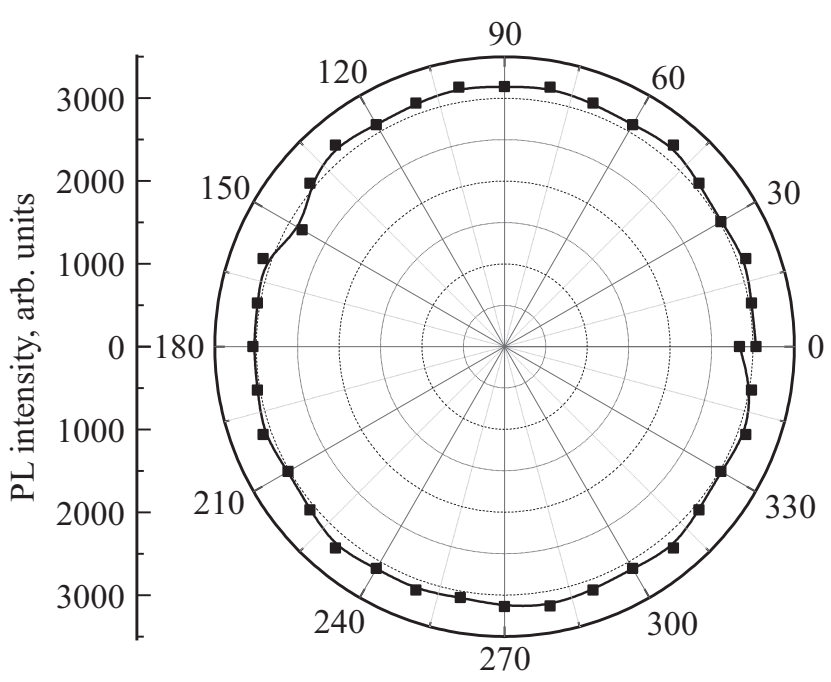

Рис. 3. Зависимость интенсивности сигнала ФЛ из КТ, находящейся внутри лежащей нанонити, от ориентации линейного поляризатора.

что излучение из боковой поверхности нанонити при рекомбинации экситона в квантовой точке практически не поляризовано (рис. 3). Это выглядит странно, так как для квантовой точки, диаметр которой много больше ее толщины, естественно было бы ожидать, что основное состояние экситона связано с тяжелой дыркой. В этом случае экситонная ФЛ из квантовой точки должна быть линейно поляризована перпендикулярно оси нити со степенью поляризации в 100\%. Однако в наших образцах наблюдается либо слабая поляризация, либо ее отсутствие.

Оба этих явления приводят к загадочной картине: в тех случаях, когда ожидалось, что сигнал ФЛ будет линейно поляризован, поляризация отсутствовала, а когда ожидалось отсутствие поляризации, сигнал ФЛ был поляризован.

\section{4. Обсуждение}

Наблюдаемую поляризацию люминесценции КТ из нанонити с точки зрения симметрии можно было бы объяснить, учитывая, что квантовая точка смещена относительно оси нити. Это привело бы к некоторой анизотропии оптических свойств нашей структуры. Однако априори неясно, достаточно ли небольшого смещения КТ относительно оси нити для того, чтобы получить столь сильное влияние на поляризацию излучения. Оценки показали, что одного этого обстоятельства недостаточно. Кроме асимметрии расположения точки в нити, необходимо учесть большой контраст диэлектрических проницаемостей нити $(\varepsilon \sim 10)$ и окружающего ее воздуха. Это приводит к появлению потенциала изображения, который может существенно увеличить энергию связи экситона.

Для оценки энергии связи экситона в наших структурах мы провели измерения спектров ФЛ из квантовой точки в магнитном поле, направленном вдоль оси нанонити. На рис. 4 представлен набор спектров ФЛ из одиночной квантовой точки, заключенной внутри нанонити в магнитных полях от -8 до +8 Тл. $\mathrm{B}$ спектре наблюдаются линии излучения экситона с энергией 1.652 эВ и биэкситона с энергией 1.643 эВ. Обе линии в магнитном поле показывают диамагнитный сдвиг и зеемановское расщепление. Величина зеемановского расщепления практически одинакова для экситона и биэкситона и составляет около 1 мэВ в поле 8 Тл, что соответствует величине $g$-фактора $\sim 1.0$. Коротковолновый (диамагнитный) сдвиг этих линий составляет 0.5 мэВ в поле 8 Тл, т.е. величина диамагнитного сдвига не превосходит 8 мкэВ/Т ${ }^{2}$. Это примерно на порядок меньше величин диамагнитного сдвига в квантовых ямах GaAs/AlGaAs [4]. Сравнивая данные о величинах диамагнитного сдвига и энергии связи экситона, приведенные в работе [4], для квантовых ям и наши данные, мы можем сделать вывод о том, что энергия связи экситона в нашем случае должна находиться в диапазоне 50-80 мэВ. Столь большая величина энергии связи экситона в нанонитях была ранее предсказана в теоретической работе [5]. В этой работе показано, что учет потенциала изображения при большом различии диэлектрических проницаемостей нити $(\varepsilon=12.8)$ и окружающего ее воздуха $(\varepsilon=1)$ приводит к росту энергии связи экситона в тонких нитях GaAs вплоть до величины в 0.8 эВ. Для диаметра нити соответствующей нашим образцам, приведенный в работе [5] расчет дает величину порядка 70 мэВ, что согласуется с приведенным выше диапазоном значений, определенным экспериментально. Спектр, представленный на рис. 4, позволяет также измерить величину энергии связи биэкситона, которая составляет 8.5 мэВ, что примерно в 2 раза больше известных значений этой величины в КT GaAs/AlGaAs, выращенных методом самоорганизации [6]. Большая энергии связи биэкситона хорошо согласуется с выводом о большей величине энергии связи самого экситона, поскольку определяется в существенной степени последней.

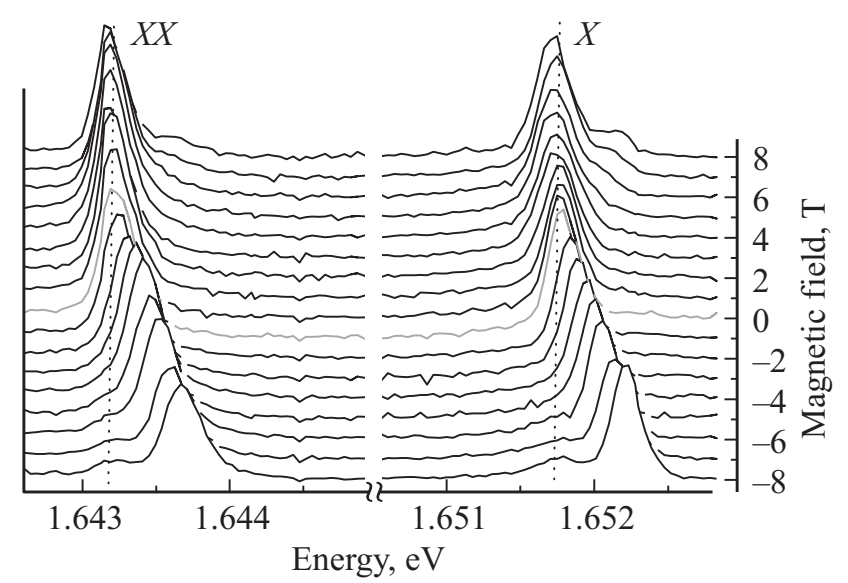

Рис. 4. Спектры циркулярно-поляризованной ФЛ экситона и биэкситона из КТ, помещенной в нанонить при различных магнитных полях от -8 до +8 Тл. Геометрия Фарадея. Величина поля каждого спектра отмечена на правой шкале. $T=1.5 \mathrm{~K}$. 


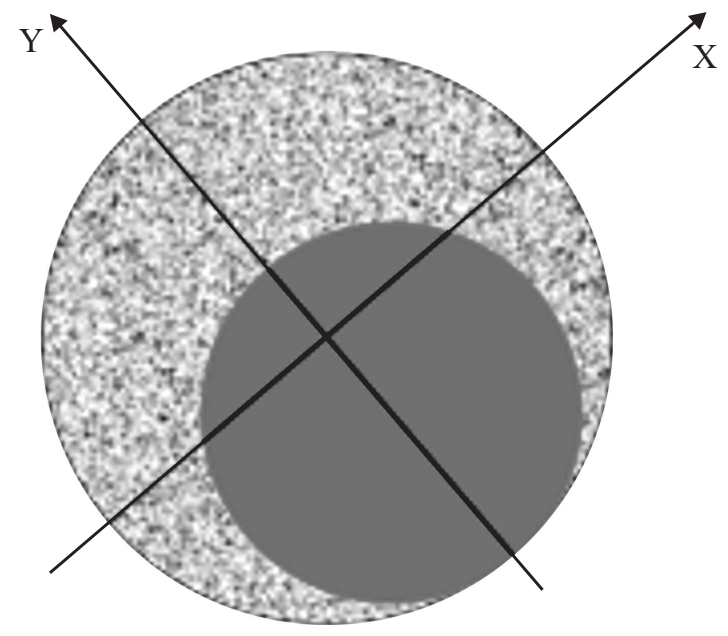

Рис. 5. Схема неаксиального расположения КТ внутри нанонити в сечении, перпендикулярном оси роста. Серый цвет соответствует нанонити, темно-серый - КТ.

Заметим, что энергия расщепления легких и тяжелых дырок при их независимом квантовании в КТ составляет величину $\sim 15$ мэВ, т. е. много меньше, чем измеренная энергия связи экситона. Это означает, что потенциал изображения должен приводить к сильному смешиванию состояний легких и тяжелых дырок. Таким образом, в наших КТ основное состояние экситона определяется линейной комбинацией волновых функций состояний легких и тяжелых дырок [7].

Если при таком гигантском влиянии диэлектрического контраста на энергию связи экситона сама квантовая точка окажется смещенной от оси нити даже на небольшую величину в 5-10\% от ее диаметра, то смешивание легких и тяжелых дырок приведет к появлению линейной поляризации излучения при рекомбинации экситона (рис. 5). Подобное явление наблюдалось для экситонов, локализованных на анизотропном интерфейсе $[8,9]$. Кроме того, смешивание состояний легких и тяжелых дырок в экситоне может привести к появлению излучения, поляризованного как поперек, так и вдоль оси нанонити.

\section{5. Заключение}

Исследованы спектры фотолюминесценции полупроводниковых структур, представляющих собой квазиодномерные нити на основе $\mathrm{Al}_{0.3} \mathrm{Ga}_{0.7} \mathrm{As}$. Диаметр типичной нанонити составлял 20-50нм, а длина 0.5-1 мкм. В центре каждой квантовой нити находилась одна или несколько квантовых точек на основе GaAs. Толщина точки составляла 2 нм, а диаметр 20-40 нм. Спектры фотолюминесценции снимались при разных интенсивностях оптического возбуждения в магнитных полях до 8 Тл. В спектрах одиночной квантовой точки наблюдались линии экситона и биэкситона. Эти линии быстро насыщались с ростом интенсивности возбуждения. В магнитном поле эти линии расщеплялись с $g$-фактором $\sim 1$ и испытывали диамагнитный сдвиг величиной $\sim 0.5$ мэВ в поле 8 Тл. Это позволило оценить энергию связи экситона в нашей структуре в диапазоне 50-80 мэВ. Обнаружено, что сигнал ФЛ, наблюдаемый в направлении [111], линейно поляризован в направлении [110]. Эффект объясняется неаксиальным расположением квантовой точки относительно оси нити.

А.В. Платонов и В.П. Кочерешко благодарят РФФИ (грант № 14-02-01244) за частичную финансовую поддержку.

\section{Список литературы}

[1] В.П. Кочерешко, В.Н. Кац, А.В. Платонов и др. Поверхность, 9, 18 (2012).

[2] V.N. Kats, V.P. Kochereshko, A.P. Platonov et al. Semicond. Sci. Technol., 27 (1), 150091 (2012).

[3] А.В. Платонов, В.П. Кочерешко, В.Н. Кац и др. Поверхность, 7, 20 (2013).

[4] G. Duggan. Phys. Rev. B, 37, 2759 (1988).

[5] Е.А. Муляров, С.Г. Тиходеев. ЖЭТФ, 111, 274 (1997).

[6] M. Abbarchi, T. Kuroda, T. Mano et al. Phys. Rev. B, 82, $201301 \mathrm{R}(2010)$

[7] М.А. Семина, Р.А. Сурис. ФТП, 49, 817 (2015).

[8] Е.Л. Ивченко, А.А. Торопов, П. Вуазен. ФТТ, 10, 1925 (1998).

[9] А.С. Гуревич, В.П. Кочерешко, А.В. Платонов и др. ФТТ, 4, 759 (2004).

Редактор А.Н. Смирнов

\section{The photoluminescence polarization of the quantum dots embedded into the quantum wires}

\author{
A.V. Platonov*, V.P. Kochereshko*+, V.N. Kats*, \\ G.E. Cirlin*, A.D. Bouravleuv*, A. Delga $\bullet$, \\ L. Besombes ${ }^{\bullet}$, H. Mariette ${ }^{\bullet}$ \\ * loffe Institute, \\ 194021 St. Petersburg, Russia \\ + Saint Petersburg State University, \\ 199034 St. Petersburg, Russia \\ - CEA-CNRS group „Nanophysique \\ et Semiconducteurs", \\ CEA, INAC, SP2M, and Institut Néel, \\ 17 rue des Martyrs, F-38054 Grenoble, France
}

\begin{abstract}
The photoluminescence spectra of the single quantum dot embedded into the quantum wire have been studied. Using the spectra obtained in a magnetic field we were able to estimate the binding energy of an exciton in the quantum dot enclosed into the quantum wire. It has been found also that the exciton photoluminescence signal propagating along the wire was linearly polarized. At the same time for the light propagating perpendicular to the wire growth axes the photoluminescence signal was virtually not polarized. The observed effect is explained by assuming nonaxial arrangement of the dot within the wire and taking into account a giant increase of the exciton binding energy associated with dielectric contrast between the wire material and surrounding media.
\end{abstract}

